\title{
Listeners judge talker sex more efficiently from male than from female vowels
}

\author{
MichaEL J. OWREN \\ Georgia State University, Atlanta, Georgia \\ Michael BeRKOWITZ \\ Cornell University, Ithaca, New York \\ AND \\ JO-ANNE BACHOROWSKI \\ Vanderbilt University, Nashville, Tennessee
}

\begin{abstract}
Speech routinely provides cues as to the sex of the talker; in voiced sounds, these cues mainly reflect dimorphism in vocal anatomy. This dimorphism is not symmetrical, however, since during adolescent development, males specifically diverge from a previously shared trajectory with females. We therefore predicted that listeners would show a corresponding perceptual advantage for male sounds in talker-sex discrimination, a hypothesis tested using very brief, one- to eight-cycle vowel segments. The expected performance asymmetry was observed in threshold-like tests of multiple different vowels in Experiments 1-3, and a signal detection design in Experiment 4 helped rule out possible response bias effects. In confirming our counterintuitive prediction, the present study illustrates that a biological and evolutionary perspective can be helpful in understanding indexical cuing in speech.
\end{abstract}

Researchers have long noted that in addition to its linguistic value, speech provides salient acoustic cues as to talker characteristics, including biological sex, individual identity, emotional state, and more. Although they are widely acknowledged to be important, these "personal" or "indexical" cues (see, e.g., Abercrombie, 1967; Ladefoged, 1967) have received less empirical attention than have the linguistic components (see, e.g., Goldinger, Pisoni, \& Luce, 1996; Johnson \& Mullennix, 1997; but see Hollien, 2002; Rose, 2002). Understandably, investigators have emphasized the question of how listeners recover linguistic information in the face of variable speech acoustics, rather than focusing on that variability itself. Work on indexical cuing has therefore tended to be pragmatic rather than theoretically inspired - for instance, in its orientation toward improving speech synthesis or automatic speech recognition (e.g., Childers \& Wu, 1991; Wu \& Childers, 1991; but see Fitch, 2000). However, indexical aspects of speech can also play a crucial role in understanding phonetic content (e.g., Lachs, McMichael, \& Pisoni, 2000; Pisoni, 1997), and it is important both to document such cues and to develop a stronger theoretical understanding of their occurrence and form.

Bachorowski and Owren (1999) suggested adopting an explicitly biological approach to indexical cuing of talker sex and individual identity, arguing that naturally occurring anatomical variation is the most basic and stable source of cues to these traits. If so, perception of talker sex in particular should first and foremost mirror the audible correlates of anatomical dimorphism, which occur species-wide in humans. Aspects of this approach are implicit in previous investigations of talker-sex perception (e.g., Bennett \& Montero-Diaz, 1982; Coleman, 1976; Lass, Hughes, Bowyer, Waters, \& Bourne, 1976) and can be elaborated more explicitly by combining principles of sexual selection with the source-filter theory of speech production (e.g., Fant, 1960).

\section{Vocal Production, Sexual Selection, and Anatomical Dimorphism}

Voiced or phonated sounds are of particular interest in this context - that is, those sounds whose source energy is created through regular vocal-fold vibration that gives rise to a rich set of harmonics. The fundamental frequency of vocal-fold vibration $(f 0)$ largely determines the perceived pitch of such sounds, whereas vocal-tract cavities above the larynx also leave a characteristic "imprint" on the sound's frequency spectrum through their shape- and size-specific filtering properties. This supralaryngeal vocal-tract transfer function is modeled as a series of resonances (formants) and produces characteristic patterns of high- and low-energy harmonics across the frequency spectrum.

Investigators interested in indexical features have shown that unambiguous acoustic correlates of an adult

M. J. Owren, owren@gsu.edu 
talker's sex occur in both $f 0$ and formant frequencies (e.g., Bachorowski \& Owren, 1999; Childers \& Wu, 1991; Hillenbrand, Getty, Clark, \& Wheeler, 1995; Peterson \& Barney, 1952; Rendall, Kollias, Ney, \& Lloyd, 2005; Wu \& Childers, 1991) and that both adults (e.g., Coleman, 1976; Lass et al., 1976; Whiteside, 1998a) and children (Bennett \& Montero-Diaz, 1982) can readily distinguish whether adult talkers are male or female. Both kinds of cues are traceable to sexual dimorphism occurring in the source and filter components of adult vocal production anatomy (Fant, 1966; Titze, 1994). For example, the vibrating segments of the vocal folds are approximately $60 \%$ longer in adult males than in adult females (Titze, 1989), and vocal-tract length is approximately $15 \%-20 \%$ greater in males (Fant, 1966; Goldstein, 1980). Male vowel sounds requisitely show lower-frequency $f 0$ and formant values than do female versions, and listeners are likely to be able to hear a talker's sex reliably by attending to either dimension.

However, vocal-tract differences can have more subtle implications as well. Dimorphism can originate from sexual selection, which occurs when individuals of the same sex compete for access to opposite-sex mates (intrasex competition), compete to be selected as a mate by members of the opposite sex (mate choice competition), or both. In humans, sexual selection does not create male-female differences in primary sexual characteristics such as genitalia, but instead is associated with dimorphism in secondary sexual characteristics such as body size, body fat distribution, facial morphology, and voice attributes (these findings are reviewed by Boyd \& Silk, 2000). In these cases, each sex has acquired distinctive and unique features that need not have direct counterparts in the other.

In humans, puberty produces marked changes in male voices, creating features that may function in both intrasex and mate choice competition (see, e.g., Collins, 2000; Feinberg, Jones, Little, Burt, \& Perrett, 2005; Fitch, 2000; Puts, 2005; Puts, Gaulin, \& Verdolini, 2006). Vocal-fold and vocal-tract lengths do in fact increase significantly in both sexes over the course of development, but male puberty and adolescent growth in particular are associated with dramatic laryngeal enlargement and vocal-tract elongation (see, e.g., Fitch \& Giedd, 1999; Perry, Ohde, $\&$ Ashmead, 2001; Whiteside, 2001). Thus, although the developmental trajectories shown by boys and girls are subtly different before puberty (reviewed by Whiteside, 2001), these differences are dwarfed by the dramatic divergence that occurs as human adolescents become mature men and women. Whereas development in females continues virtually unperturbed into adulthood, $f 0$ values in males abruptly fall to approximately $50 \%-60 \%$ of those of females, and male formant frequencies decline to approximately $80 \%-90 \%$ of female versions (estimates based on Bachorowski \& Owren, 1999; Hillenbrand et al., 1995; Peterson \& Barney, 1952; Rendall et al., 2005).

\section{Talker-Sex Perception}

Noting that human vocal ontogeny is asymmetrical leads to a counterintuitive prediction concerning auditory perception of talker sex. Specifically, because sexual selection leads males to diverge from the "default" female form, adult male voices can be considered "marked" by the sexually selected features of lowered $f 0$ and formant frequencies. It therefore follows that listeners should hear talker sex somewhat more easily in male than in female voiced sounds. Specifically, the presence of critical features of "maleness" virtually guarantees that the talker is an adult male. However, their absence does not unequivocally imply that the talker is an adult female. The individual might, for instance, be a young male whose voice has not yet changed, or a postpubertal male whose vocal tract has not diverged as far from the female form as is typically the case. This prediction of asymmetry is counterintuitive, in that the perceptual task itself involves two mutually exclusive choices and is, in that sense, entirely symmetrical. However, if sexual selection and its effects on male vocal-tract ontogeny are taken into account, the presence of low-frequency $f 0$ and formants becomes critically disambiguating.

Previous studies. Earlier work has shown that listeners are readily able to judge talker sex from short speech segments, and these findings provide some evidence for the predicted discrimination asymmetry. For example, Lass et al. (1976) tested 15 listeners with six sustained vowels, both voiced and whispered, recorded from 10 male and 10 female talkers. Listeners heard a 3-sec vowel segment on each trial and reported whether the talker was male or female. Their overall accuracy was high- $96 \%$ correct across 1,800 judgments with voiced vowels, 75\% with whispered vowels, and $91 \%$ with vowels that were low-pass filtered at $255 \mathrm{~Hz}$ to isolate the $f 0$ component. Although possible sex differences were not tested statistically, Lass et al. also found that across all three conditions and for each vowel, listeners were more accurate when classifying male sounds than when classifying female sounds. With voiced versions, accuracy with the male stimuli ranged from $96.5 \%-100 \%$ correct across the various vowels, and dropped to $87.0 \%-97.5 \%$ correct for female vowels.

Coleman (1971) tested talker-sex classification performance with vowel-like samples that 10 male and $10 \mathrm{fe}-$ male talkers produced using a laryngeal vibrator fixed at $85 \mathrm{~Hz}$. This approach effectively removed $f 0$-related cues, in that both sexes produced the sounds using the same source energy. Talkers were recorded as they produced sustained $/ \mathrm{i} /$ - and $/ \mathrm{u} /$-like sounds that were then presented to 15 listeners as 2.5 -sec segments. A total of 265 of 300 sounds were classified correctly by sex ( $88 \%$ correct), with male samples misclassified in only 3 of 150 cases ( $98 \%$ correct) and female samples misclassified in 32 of 150 cases ( $79 \%$ correct). Coleman (1976) later extended this work, using a laryngeal vibrator that could produce both $120-$ and $240-\mathrm{Hz}$ signals. In the most pertinent experiment, 25 listeners heard vowels produced by 5 female talkers who by design had relatively high formants and by 5 males that had relatively low formants. Several stimulus types were tested, the most important for present purposes being the most naturalistic case, in which $f 0$ values were matched to the sex of the talker. In this condition, listeners were $98 \%$ correct over 250 total trials, with the five errors that did occur involving only female talkers. 
Talker-sex perception in adults has been examined in a number of other studies as well (e.g., Ingemann, 1968; Schwarz, 1968; Schwarz \& Rine, 1968; Traunmüller, 1997; Whiteside, 1998a, 1998b), and Bennett and Montero-Diaz (1982) have provided evidence that children may also exhibit a performance asymmetry in discriminating talker sex through voiced vowels. These researchers tested a total of 84 children (ages 6-8 years) in one of four conditions involving voiced or whispered /æ/ sounds produced either by adults or by other children. Overall listener accuracy was $93.6 \%$ correct for vowels from adult males, showing a statistically significant decline to $84.6 \%$ correct for vowels from adult females. Identification of voiced vowels was virtually perfect, with the children showing $100 \%$ versus $97.4 \%$ correct for male and female voices, respectively. The participants were less accurate with whispered vowels, and a stronger difference emerged for male versus female talkers- $86.4 \%$ versus $71.2 \%$ correct, respectively.

Present design. The work reported here sought to test the hypothesis that because male-female differences in human vocal anatomy are grounded in asymmetrical developmental trajectories, listeners should show a corresponding perceptual asymmetry in distinguishing a talker's sex from voiced speech. Under normal circumstances, any such effect is almost certain to be obscured by the general abundance and salience of talker-sex cues in speech. We nonetheless expected that if the discrimination task could be made sufficiently challenging, listeners should be at least slightly and reliably more efficient in hearing talker sex from male than from female vowels. Our experimental strategy was conceptually very simple - namely, to present vowel stimuli that were brief enough to create uncertainty about the talker's sex, and thereby reveal the predicted performance asymmetry as fully and reliably as possible. Although earlier studies provided evidence of better performance with male vowels even with sustained sounds (e.g., Coleman, 1971, 1976; Lass et al., 1976), the magnitude of the asymmetry has been variable, and the reliability of the difference has not been subject to systematic testing.

Results reported by Whiteside (1998a) suggested that the vowel segments used would likely have to be extremely short in order to fully reveal any possible performance asymmetry. In Whiteside's (1998a) experiment, 3 listeners discriminated the sex of talkers using short segments of vowels produced by 3 different males and 3 different females. Stimuli were either 100 or $50 \mathrm{msec}$ long, and the participants achieved an accuracy rate of $98.9 \%$ correct for 60 different sounds. In light of these findings, our approach was to test with literally the shortest vowel segments from which listeners could possibly perform the task, meaning sounds whose short durations would put them as close as possible to the "threshold of detection" for hearing talker sex. It was not known where that threshold would be, however, or where the predicted performance asymmetry might emerge relative to that point. Our first three experiments were therefore based on the classical psychophysical method of constant stimuli (Gescheider, 1997), a procedure in which stimuli are selected so as to range from clearly below to clearly above threshold. We anticipated that the predicted performance asymmetry would most likely emerge at durations at which listeners were relatively close to the limits of discrimination, while nonetheless performing at above-threshold levels.

Creating very brief vowel segments required a choice between using stimuli in which male and female versions were equated for temporal duration or for number of cycles. As cyclically based sounds, vowel waveforms are not readily amenable to editing at arbitrary points. The stimuli used were therefore always based on complete cycles, with exact duration becoming secondary to the number of cycles included. However, because $f 0$ values are approximately twice as high in females as in males, there are of course approximately twice as many cycles per unit of time in a female vowel than in a male vowel. In Experiments 1-3, stimuli were equated for number of cycles, which created the confound that male vowel segments were almost twice as long as the corresponding female segments. The opposite tack was taken in Experiment 4, which utilized a signal detection design rather than the method of constant stimuli. Here, male stimuli were based on twice as many cycles as corresponding female versions, thereby producing approximately equal stimulus durations across talker sexes.

The first three experiments showed listeners to be very sensitive to talker-sex cues, producing an overall discrimination threshold of 1.7 cycles or less. Each experiment also revealed evidence of the predicted performance asymmetry. Experiment 1 tested listeners with /e/ vowels, Experiment 2 replicated the findings using the point vowels $/ \mathbf{i} /, / \mathbf{u} /$, and $/ \mathrm{a} /$, and Experiment 3 replicated the effect using reduced vowel sounds in order to help rule out the possibility that the asymmetry was due to females having a larger vowel space than males. In the fourth experiment, listeners heard pairs of equal-duration stimuli that were either both from the same sex or from different sexes, and they then responded whether the sounds were the "same" or "different" on that basis. This experiment also produced a robust performance asymmetry effect, as well as helping to rule out two alternative possibilities, either that the asymmetry was due to having equated for number of cycles in the earlier experiments or that listener hit rates for male vowels had been inflated by an unintentional bias to respond "male" to ambiguous stimuli.

\section{GENERAL METHOD}

\section{Participants}

The participants were undergraduates working for course credit. All were native English speakers who reported having no speech or hearing impairments. Participants provided informed consent and completed a personal history questionnaire before the session, and they were debriefed immediately after the session.

\section{Materials}

Apparatus. The participants were tested in a small room containing five booths equipped with Beyerdynamic DT831 headphones (Farmingdale, NY) and four-button response boxes (Tucker-Davis Technologies [TDT], Alachua, FL). Each booth was connected to TDT control modules in an adjacent room that were operated by computer using custom-written software (by B. Tice and T. Carrell, available at hush.unl.edu/LabResources.html). Stimuli and support files were prepared using this software in conjunction with the 

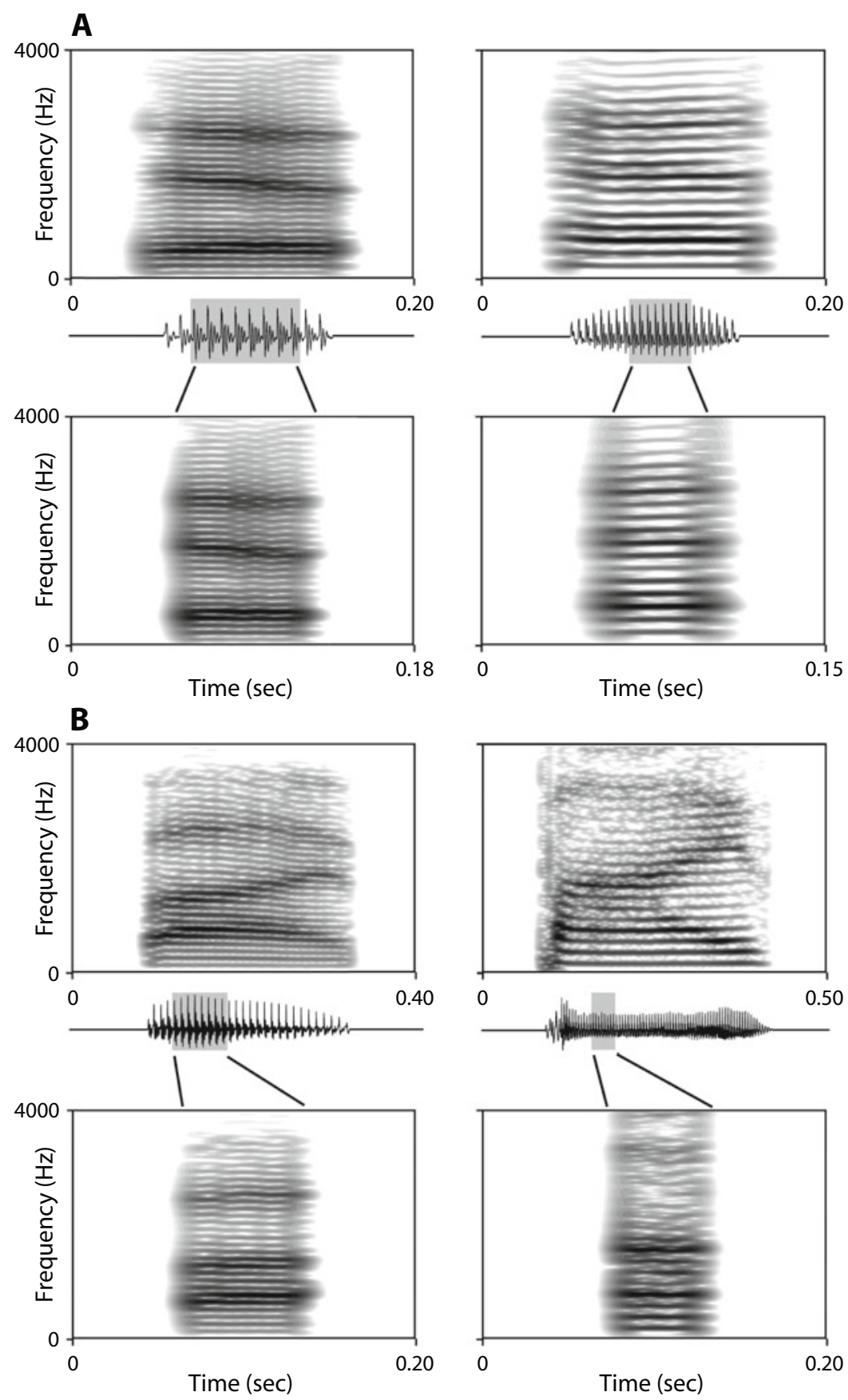

Figure 1. Waveforms and narrow-band spectrograms (0.03-sec Gaussian analysis window) of four of the stimuli used in the experiments. (A) / $/$ / vowels excised from the word "test." (B) /a/ sounds excised from the / $\mathrm{a}^{\mathrm{I}} /$ phoneme of the word "five." Male versions are shown on the left, and female versions on the right. Spectrograms immediately above the waveforms illustrate full-length versions and those immediately below show eight-cycle stimulus segments corresponding to the shaded areas of the waveforms.

acoustics programs Praat 4.15 (Boersma, 2001), ESPS/waves + 5.3 (Entropic Research, Washington, DC), and SpeechStation II (Sensimetrics, Cambridge, MA). Statistical analyses were conducted with NCSS (Jerry L. Hintze, Kaysville, UT).

Stimuli. Stimuli were based on single vowel segments from either 5 or 6 talkers of each sex. Multiple stimuli were derived from each token, as illustrated in Figure 1 for the sounds $/ \varepsilon /$ and $/ a /$ (the latter of which was excised from the diphthong $/ \mathrm{a}^{\mathrm{I}} /$ ). A finished stimulus consisted of either a single sound (Experiments 1-3) or a pair of sounds (Experiment 4). To avoid audible transients during presentation, all stimuli began with a silent interval of up to $100 \mathrm{msec}$ and ended with $100 \mathrm{msec}$ of silence.

Stimuli consisted of one, two, three, four, five, six, or eight cycles. One-cycle stimuli were always created first and were based on the 

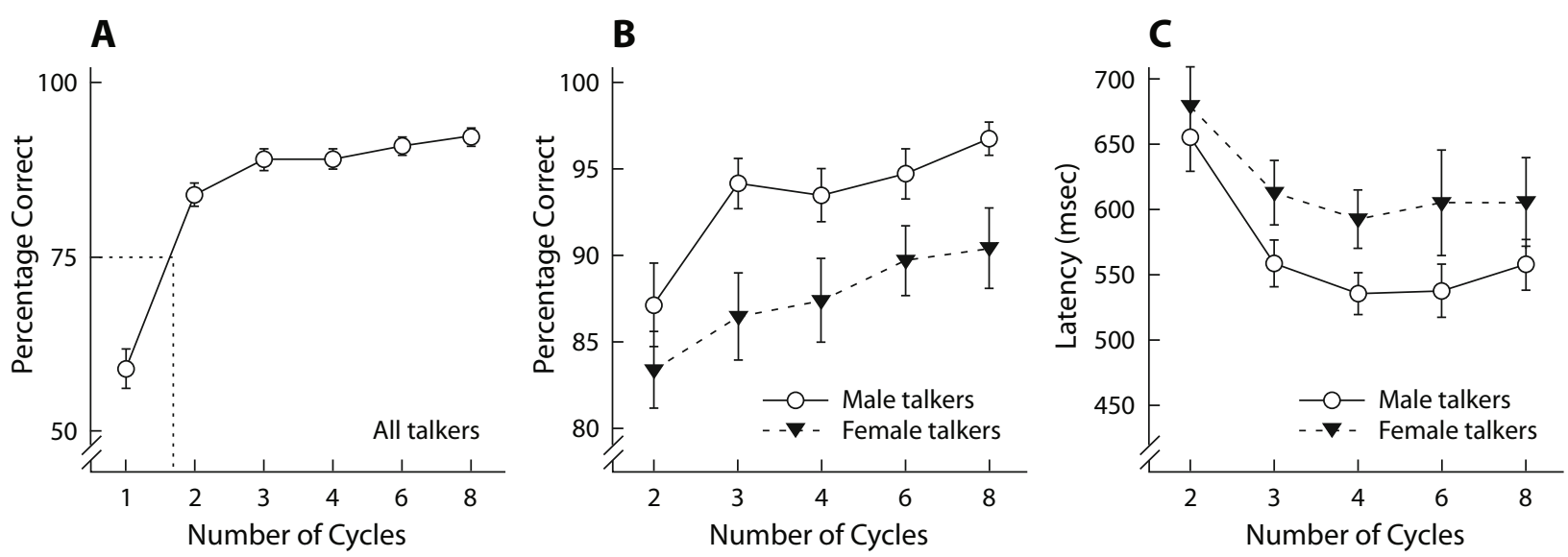

Figure 2. Response data from Experiment 1 with $/ \varepsilon /$ sounds. (A) Percentage correct means and standard errors by number of cycles, as well as the threshold value corresponding to $75 \%$ correct. (B) Percentage correct means and standard errors for two- to eight-cycle stimuli, by talker sex. (C) Latency means and standard errors for two- to eight-cycle stimuli, by talker sex. Some standard error bars are too small to be clearly visible.

highest-amplitude cycle occurring near the midpoint of a sound. The other stimuli were centered on this cycle, but included in addition the next cycle, the two bracketing cycles, and so on.

\section{Procedure}

Participants were tested in groups of up to 5. Their instructions included the facts that the stimuli consisted of a series of vowel sounds produced by males and females, that each trial would be cued by lights on the response box, and that responding should occur as quickly as possible using labeled buttons on the box. Label position was reversed daily for counterbalancing purposes. The participants responded using their two index fingers, resting them lightly on the buttons at all times. Before beginning the experiment, listeners completed practice trials with stimuli that were comparable to those used in the experimental session but were from different talkers than the ones represented in the experimental stimuli. Stimulus order was randomized in each session, and trial parameters included a 4,000-msec response window and 2,000-msec intertrial interval. Response latencies were measured from the onset of either the first and only sound in the file (Experiments 1-3) or from the onset of the second sound (Experiment 4). Sessions lasted about $40 \mathrm{~min}$.

\section{Data Analysis}

Dependent variables in Experiments 1-3 were the mean percentages correct and median response latencies by condition. Discrimination thresholds were calculated separately by individual listener as the interpolated number of cycles corresponding to $75 \%$ correct. This particular value is often used as a performance criterion in two-choice psychophysical discrimination, because it represents the midpoint between chance responding and perfect performance (Gescheider, 1997). In Experiment 4, vowel segments were presented in pairs on each trial in a same-different signal detection design.

Statistical analyses emphasized repeated measures ANOVA testing, with listener sex as a between-subjects variable, talker sex and number of cycles as within-subjects variables, and listener as the subjects variable. Newman-Keuls analyses were used for post hoc comparisons, which focused on differences between stimuli with varying numbers of cycles.

\section{EXPERIMENT 1}

\section{Method}

Participants. There were 24 participants ( 12 males, 12 females).

Stimuli. Sounds were selected from a database of $/ \varepsilon /$ vowels taken from the phrase "test $n$ test," recorded from participants in a computer-based lexical-decision task (Bachorowski \& Owren, 1995). The " $n$ " segment of this repeated announcement ranged from "one" to "ten" for baseline trials and from "two" to "twenty-two" over the experimental session. The stimuli used here were based on a single $/ \varepsilon /$ from each of 6 males and 6 females, as shown in Figure 1A.

Six stimuli were derived from each token, consisting of one, two, three, four, six, or eight cycles, in order to create a total of 72 different sounds. The vowels originally included approximately 13 cycles for male talkers and 25 cycles for female talkers. Stimulus loudness was found to grow somewhat as the number of cycles increased, potentially affecting the determination of threshold values, and therefore two versions of each stimulus were used. Equal-amplitude versions were all set to a mean of $80 \mathrm{~dB}$. Adjusted-amplitude sounds were subjectively matched in loudness by one of the experimenters, with two-cycle stimuli set to $77 \mathrm{~dB}$, three- and four-cycle stimuli set to $75 \mathrm{~dB}$, and six- and eight-cycle stimuli set to $72 \mathrm{~dB}$.

Procedure. Before beginning the experiment, listeners completed 24 practice trials that included all stimulus versions from 1 male and 1 female talker. These trials exposed participants to the full range of stimulus lengths, with male and female sounds alternating systematically in ascending order of the number of cycles involved. The experimental session included 72 sounds and two amplitude levels, for a total of 144 trials.

\section{Results and Discussion}

Discrimination performance. Across analyses, there were no main effects of listener sex on percentages correct or latencies, and this factor will not be considered further. Overall mean percentage correct outcomes by number of cycles are shown in Figure 2A. The mean threshold value across all listeners was 1.70 cycles $(S E=0.10)$, and there was no effect of the equal-amplitude versus adjustedamplitude manipulation on this value. Overall performance with one-cycle stimuli ( $M=58.3 \%, S E=2.98)$ was found to be higher than the chance rate of $50 \%$ [one-sample $t$ test: $t(23)=3.85, p<.001]$ but below the threshold of $75 \%$ $[t(23)=-7.74, p<.0001]$. Repeated measures ANOVA testing of the overall percentage correct across all stimuli revealed strong effects of number of cycles $[F(5,110)=$ $82.8, p<.0001]$. Post hoc comparisons showed that response accuracy was significantly lower for one-cycle stimuli than for the others, that two-cycle sounds $(M=$ 
$84.5 \%, S E=1.71)$ were classified significantly less reliably than longer versions, and that the longer versions did not differ among themselves $(M=91.2 \%, S E=0.72)$.

The pattern was thus below-threshold performance with one-cycle stimuli, intermediate but above-threshold accuracy with two-cycle sounds, and better, undifferentiated performance with the others. These outcomes were mirrored in the latency data, which showed a main effect of number of cycles and corresponding statistically significant post hoc comparisons.

Performance asymmetry. There was no main effect of talker sex, but comparisons that included all cycles did reveal a talker sex $\times$ number of cycles interaction $[F(5,110)=38.0, p<.0001]$. This interaction was due to a talker-sex-based asymmetry occurring in the two- to eight-cycle stimuli. Restricting analysis to these abovethreshold stimuli revealed statistically significant main effects of talker sex on both percentages correct $[F(1,22)=$ $10.1, p<.01]$ and latencies $[F(1,22)=9.82, p<.01]$. As illustrated in Figures $2 \mathrm{~B}$ and $2 \mathrm{C}$, classification of male vowels was 6.5 percentage points higher and $59.4 \mathrm{msec}$ faster than classification of female vowels.

This difference does not appear to be due simply to the fact that male stimuli were approximately twice as long as their female counterparts. Main effects remained when stimulus duration was entered as a covariate in a general linear model ANOVA, including effects on both percentages correct $[F(1,381)=6.49, p=.05]$ and latencies $[F(1,381)=6.44, p<.05]$. Furthermore, comparing percentages correct for the 24.9-msec, three-cycle male versus the 26.9-msec, six-cycle female sounds also showed a significant advantage for the male versions $[t(94)=1.75$, $p<.05$, one-tailed], although no latency difference was found. Percentage correct outcomes were not different for the 32.9-msec, four-cycle male versus the 35.7-msec, eight-cycle female stimuli, but there was a significant latency difference $[t(94)=1.86, p<.05$, one-tailed $]$.

Stimulus amplitude was found to affect accuracy for two- to eight-cycle vowel segments, with performance being slightly better for equal-amplitude $(M=91.3 \%, S E=0.83)$ than for adjusted-amplitude $(M=88.5 \%, S E=1.07)$ sounds $[F(1,22)=6.83, p<.05]$. However, there were no latency differences between the equal-amplitude $(M=611 \mathrm{msec}$, $S E=13.8)$ and the adjusted-amplitude $(M=599 \mathrm{msec}$, $S E=11.1)$ versions $[F(1,22)=1.27$, n.s. $]$.

\section{EXPERIMENT 2}

The results of Experiment 1 revealed the predicted performance asymmetry, in which above-threshold male vowels were classified somewhat more accurately and rapidly than the corresponding female sounds, as well as showing a very low threshold for hearing talker sex. This difference could not readily be explained by the duration confound arising from basing the stimuli on cycles. However, not all comparisons across segments of comparable durations were statistically significant, and the stimulus set consisted of only / $/$ / vowels. We therefore replicated the experiment with stimuli that were based on three other sounds-namely, the "point" vowels (/i/, /u/, and /a/). A more minor consideration was that stimulus amplitude adjustments had made no difference in threshold measurements in Experiment 1, but did have a small effect on above-threshold classification performance. This manipulation was therefore dropped in order to eliminate it as a source of potential variability in participant responses. Because amplitude adjustments had been made subjectively, we deemed that the most conservative approach was to set all stimuli in Experiment 2 to equal root-meansquare (RMS) amplitude levels.

\section{Method}

Participants. There were 24 participants ( 12 males, 12 females). Data from 1 additional male were excluded because his performance never exceeded the discrimination threshold.

Stimuli. Three different vowels from the same set of talkers represented by the $/ \varepsilon /$ sounds in Experiment 1 were used, with 1 talker of each sex dropped in order to reduce the number of trials involved. The remaining 5 male and 5 female talkers were each represented by six stimuli created from each of the three different vowels, for a total of 180 sounds. The tokens used were edited from the numbers spoken in the "test $n$ test" phrase, choosing those that included the clearest examples of $/ \mathrm{i} /, / \mathrm{u} /$, and $/ \mathrm{a} /$. The first two vowels were readily obtained, with /i/ taken from "three" or any number with a "-teen" suffix, and /u/ drawn from "two" or "twenty-two." Comparable /a/ segments were not available, and were instead excised from the $/ \mathrm{a}^{\mathrm{I} /}$ diphthong found in "five," "nine," and "nineteen." This editing proved to be straightforward, since it required identifying only eight adjacent cycles of the /a/ vowel that occurred before the /i/ offglide began. The process is illustrated in Figure 1B. The stimuli again consisted of one, two, three, four, six, or eight cycles, constructed as before and set to a mean amplitude of $80 \mathrm{~dB}$.

Procedure. The instructions now noted that the stimuli included three different vowels. There were 36 practice trials consisting of all 18 stimuli from 1 male and 1 female talker who were not used in the experiment itself; the trials alternated systematically between the two in ascending order of the number of cycles involved, but separately by vowel. The procedure was otherwise the same as in Experiment 1.

\section{Results and Discussion}

Discrimination performance. The results of this study are illustrated in Figure 3 and were very similar to those of Experiment 1. There were again no main effects of listener sex on either percentages correct or latencies, and this factor will not be considered further.

The mean threshold across all listeners was $1.71(S E=$ $0.10)$, and the mean percentage correct for one-cycle stimuli $(M=59.9, S E=2.78)$ was not different from $50 \%$ $[t(23)=1.46$, n.s. $]$ but was significantly less than $75 \%$ $[t(23)=-2.22, p<.05]$. Repeated measures ANOVA testing of the percentages correct for all stimuli again showed a strong effect of number of cycles $[F(5,110)=$ 131.0, $p<.0001]$, and post hoc comparisons replicated the accuracy pattern observed in Experiment 1 (i.e., lowest at one cycle, and lower at two cycles than for the remaining stimuli). The latency data showed parallel statistical effects in this case as well.

Performance asymmetry. Although there was no overall influence of talker sex $[F(1,22)=0.46$, n.s. $]$, there was again a talker sex $\times$ number of cycles interaction $[F(5,110)=32.7, p<.0001]$. Accuracy for the two- to eight-cycle sounds improved in comparison with Experiment 1, but reaction times were longer than in the 
previous experiment. The increase in percentage correct scores was likely due to all stimuli in Experiment 2 being set to the same RMS level; in Experiment 1, this manipulation had produced percentage correct outcomes slightly higher than those for the adjusted-amplitude stimuli. A two-sample $t$ test comparing results for the two- to eightcycle equal-amplitude sounds used in the two experiments confirmed the lack of a difference in classification accuracy $[t(766)=-0.58$, n.s.]. However, there was a latency difference $[t(766)=-4.65, p<.0001]$, indicating that the increased phonetic variation in Experiment 2 slowed participant responses.

ANOVA testing of percentages correct for the two- to eight-cycle stimuli alone again showed a main effect of talker sex $[F(1,22)=43.8, p<.0001]$, with male vowels $(M=97.8 \%, S E=0.46)$ being classified more accurately than female vowels $(M=89.1 \%, S E=0.90)$. There was also a main effect of number of cycles $[F(3,66)=11.5$, $p<.0001]$ but no interaction of that factor with talker sex. Latency outcomes were similar, showing faster responding to male $(M=595.9 \mathrm{msec}, S E=9.84)$ than to female $(M=737.2 \mathrm{msec}, S E=17.4)$ vowels $[F(1,22)=20.5$, $p<.0001]$. Number of cycles also showed a main effect $[F(3,66)=3.43, p<.05]$ but no interaction. Overall, the talker-sex asymmetry emerged somewhat more strongly than in Experiment 1. For two- to eight-cycle stimuli, classification of male sounds was 8.8 percentage points higher and $141.3 \mathrm{msec}$ faster than classification of female sounds.

Entering stimulus duration as a covariate in an ANOVA testing the two- to eight-cycle stimuli again did not remove talker-sex effects, either for percentages correct $[F(1,573)=19.9, p<.0001]$ or latencies $[F(1,573)=$ 22.3, $p<.0001]$. Comparing results for the $26.9-\mathrm{msec}$, three-cycle male stimuli and the 28.5 -msec, six-cycle female sounds also showed significantly better classification of the male versions $[t(142)=2.23, p<.05$, one-tailed $]$. Latencies were also faster for male than for female stimuli $[t(142)=1.64, p=.051$, one-tailed $]$. Similarly, percentages correct were statistically higher for the $35.5-\mathrm{msec}$, four-cycle male sounds than for the 37.7-msec, eight-cycle female stimuli $[t(142)=2.50, p<.01$, one-tailed], and a significant latency advantage emerged for those stimuli as well $[t(142)=3.64, p<.001$, one-tailed $]$.

\section{EXPERIMENT 3}

The results for Experiments 1 and 2 were quite consistent, with both studies showing a talker-sex-based performance asymmetry and producing a threshold measurement of 1.7 cycles. The asymmetry was not readily attributable to the duration confound inherent to basing the stimuli on a fixed number of cycles, but might alternatively be explained on the basis of male-female differences in speech acoustics. Specifically, female speech has been reported to be more acoustically diverse than male speech-for example, vowel spaces in females have been found to be larger than in males (see, e.g., Deterding, 1997; Henton, 1995; Traunmüller, 1988; reviewed by Whiteside, 2001). In other words, if females routinely show more phonetic diversity among their vowels, listeners could have slightly more difficulty in classifying the sounds on other dimensions, such as talker sex.

Consistent with this view, male-female differences were higher in Experiment 2 than in Experiment 1, as would be expected from Whiteside's (2001) reexamination of Lee, Potamianos, and Narayanan's (1999) data on speech acoustics across age and sex. Whiteside's analysis of data from 56 adult talkers showed that male-female dispersion differences increase as vowels become more peripheral (see, e.g., Figure 6 in Whiteside, 2001). The stimuli in Experiment 2 were the point vowels $/ \mathrm{i} /, / \mathrm{u} /$, and /a/, which in fact define the periphery of a given talker's vowel space. In contrast, the stimuli in Experiment 1 were all from a single, more central vowel. The more acoustically and phonetically diverse stimulus set of Experiment 2 also elicited slower overall reaction times. On the other hand, accuracy with the two- through eight-cycle male $/ \mathrm{i} /, / \mathrm{u} /$, and /a/ stimuli in Experiment 2 was signifi-
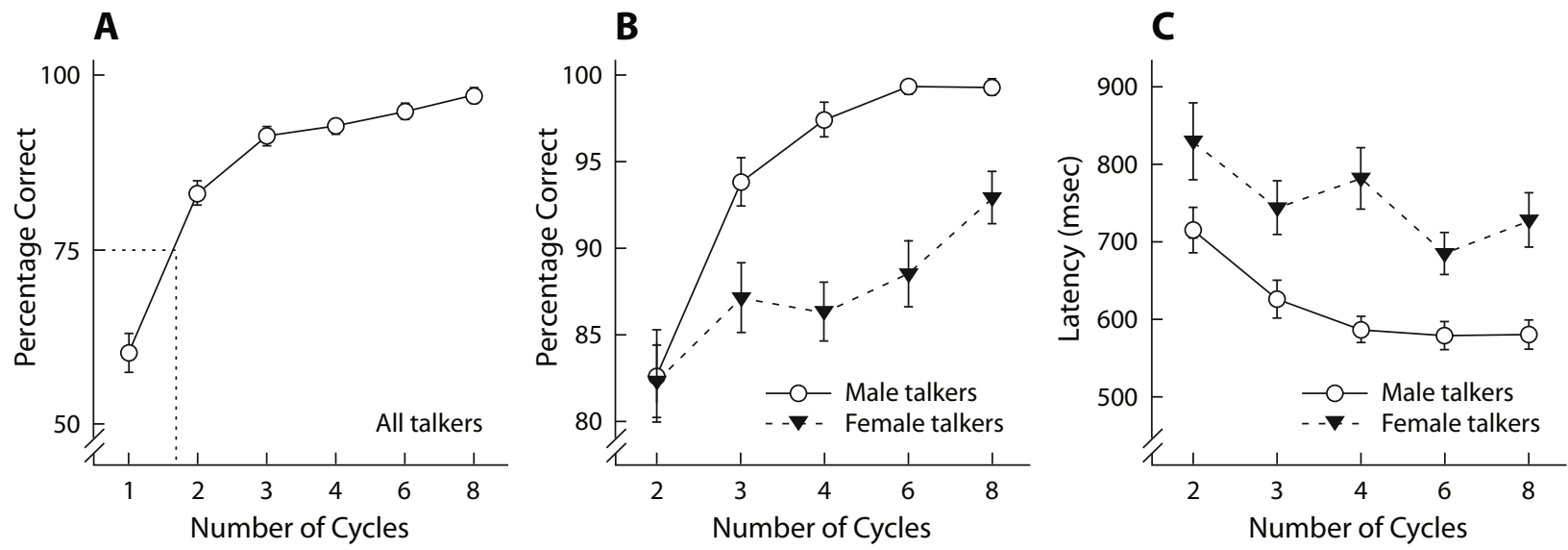

Figure 3. Response data from Experiment 2 with $/ \mathrm{i} /, / \mathrm{a} /$, and $/ \mathrm{u} /$ sounds. (A) Percentage correct means and standard errors by number of cycles, as well as the threshold value corresponding to $75 \%$ correct. (B) Percentage correct means and standard errors for two- to eight-cycle stimuli, by talker sex. (C) Latency means and standard errors for two- to eight-cycle stimuli, by talker sex. Some standard error bars are too small to be clearly visible. 

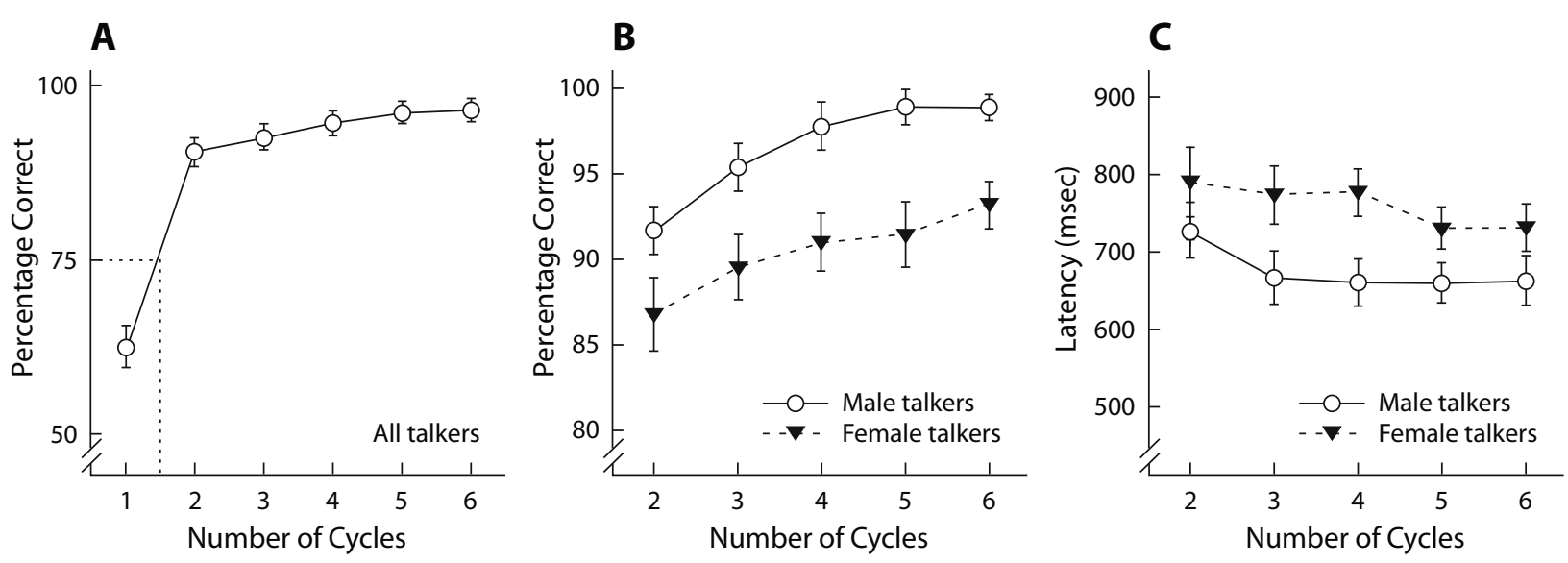

Figure 4. Response data from Experiment 3 with reduced vowel sounds. (A) Percentage correct means and standard errors by number of cycles, as well as the threshold value corresponding to $75 \%$ correct. (B) Percentage correct means and standard errors for two- to six-cycle stimuli, by talker sex. (C) Latency means and standard errors for two- to six-cycle stimuli, by talker sex. Some standard error bars are too small to be clearly visible.

cantly higher than accuracy with the corresponding female $/ \varepsilon /$ sounds in Experiment 1. This comparison suggests that male-female differences in acoustic diversity alone were not likely to be the cause of the observed performance asymmetries.

A second replication was undertaken to help settle this issue, by testing for the role of acoustic diversity, at least indirectly. In this version of the experiment, the stimuli were based on vowels that were reduced or neutralized because they appeared in unstressed word positions. We reasoned that if acoustic diversity were the critical factor in the observed performance asymmetry, the effect should be largest across the three experiments for the point vowels $/ \mathrm{i} /, / \mathrm{u} /$, and $/ \mathrm{a} /$, intermediate for the $/ \mathrm{e} /$ vowel, and smallest, or even nonexistent, for reduced (and therefore more central) vowels. Contrary to the acoustic diversity view, a perspective based on asymmetrical dimorphism predicts no notable differences for stimuli drawn from different locations of the vowel space.

\section{Method}

Participants. A total of 32 individuals (16 males, 16 females) participated as listeners, and 8 individuals ( 4 males, 4 females) participated as talkers who were the source of the reduced-vowel stimuli used in this experiment.

Apparatus. Recordings for this experiment were made using a Special Projects SP-746 head-worn microphone (Marlborough, $\mathrm{NH}$ ), connected to a $1202 \mathrm{VLZ}$ Mackie mixing console (LOUD Technologies, Woodinville, MA) and the A/D input of an SGI O2 workstation (Silicon Graphics, Mountainview, CA).

Stimuli. The 4 males and 4 females were recorded individually. Each talker read the words ahead, about, synthesis, decimal, har-

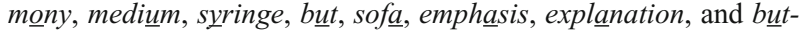
ton from a list (the target sounds are underlined). The talkers first said a word, then produced the target vowel from that word in isolation, and finally said the word again. Each individual's most suitable reduced-vowel recording was used to create stimuli that consisted of one, two, three, four, five, or six adjacent cycles. These stimuli were slightly shorter than those in the earlier experiments because the vowels occurring in unstressed positions within each word could be quite brief, particularly in male speech. The stimuli were then set to a mean amplitude of $80 \mathrm{~dB}$.
Procedure. The procedure was identical to that in Experiment 1, except that there were 16 practice trials.

\section{Results and Discussion}

Discrimination performance. The results of this study are illustrated in Figure 4, with listener performance proving to be more accurate than in either Experiment 1 or Experiment 2. Five participants performed above threshold with the one-cycle stimuli, but the remaining 27 listeners produced a mean threshold value of $1.48(S E=0.13)$. Across all participants, the mean percentage correct for one-cycle stimuli was $64.4(S E=2.41)$, which was statistically higher than $50 \%[t(31)=6.67, p<.0001]$ but also less than the $75 \%$ criterion $[t(31)=-3.70, p<.0001]$. Repeated measures ANOVA testing of percentages correct for all stimuli again showed an effect of number of cycles $[F(5,150)=78.3, p<.0001]$, and post hoc comparisons showed that performance was significantly different for one-cycle stimuli than for any other length. Performance at two cycles was also somewhat better than in Experiments 1 and 2 and could be distinguished from performance at five and six cycles, but it was not different from performance with three- and four-cycle stimuli. Accuracies with three-, four-, five-, and six-cycle stimuli were indistinguishable. The latency data were similar, with a statistically significant effect of number of cycles $[F(5,150)=27.0, p<.0001]$ and with responses to one-cycle stimuli being slower than those for the other cycle lengths, which in turn were not distinguishable from one another.

Performance asymmetry. As before, there was no overall main effect of talker sex $[F(1,30)=0.11$, n.s.], but a talker sex $\times$ number of cycles interaction did occur $[F(5,150)=31.6, p<.0001]$. Accuracy for the two- to six-cycle sounds was comparable to, or even a bit higher than, the outcomes for the two- to eight-cycle sounds in the previous experiments. In other words, in spite of being somewhat shorter (the eight-cycle sounds were in effect replaced here by five-cycle sounds), there was no dropoff in performance accuracy. 
ANOVA testing of percentages correct for the two- to six-cycle stimuli alone again showed a main effect of talker sex $[F(1,120)=20.8, p<.0001]$, with male vowels $(M=96.6 \%, S E=0.56)$ being classified more accurately than female vowels $(M=90.5 \%, S E=0.94)$. There was also a main effect of number of cycles $[F(4,120)=$ $6.46, p<.0001]$ but no interaction with talker sex. Latency outcomes were similar, showing faster responding to male $(M=694.1 \mathrm{msec}, S E=11.4)$ than to female $(M=776.6 \mathrm{msec}, S E=13.7)$ vowels $[F(1,120)=41.2$, $p<.0001]$. Number of cycles also showed a main effect $[F(3,120)=3.24, p<.05]$ but no interaction with talker sex. For classification of these stimuli, performance with male sounds was 6.1 percentage points higher and $82.5 \mathrm{msec}$ faster than performance with female sounds.

Entering stimulus duration as a covariate in an ANOVA testing the two- to six-cycle stimuli did not remove talker-sex effects, either for percentages correct $[F(1,320)=4.19$, $p<.05]$ or latencies $[F(1,320)=8.35, p<.01]$. A comparison of the $28.0-\mathrm{msec}$, three-cycle male stimuli with the 31.0-msec, six-cycle female sounds showed better classification of the male versions, a difference that closely approached statistical significance $[t(62)=1.56, p=.06$, one-tailed]. However, latencies for these sounds were statistically faster for male than for female stimuli $[t(62)=$ $3.04, p<.01$, one-tailed]. Percentages correct could not be compared for the four-cycle male sounds, since comparable, eight-cycle female sounds had not been used.

\section{EXPERIMENT 4}

The results of Experiments 1-3 indicated that across multiple different vowels, listeners were subtly but consistently better in identifying talker sex from male than from female sounds. As expected, overall performance was extremely good, revealing discrimination thresholds ranging from about 1.5 to 1.7 glottal cycles. The performance asymmetry did not appear to be due to the duration confound created by basing stimuli on numbers of cycles, nor was it critically affected by differences in the degree of articulation involved in producing the particular vowel being tested. The latter finding argues against the possibility that the larger vowel spaces and concomitantly more extreme articulatory positions reported for female talkers are important for talker-sex-based performance differences.

We nonetheless conducted a fourth experiment, for two reasons. The first was to address the confound of unequal stimulus duration through a direct test. The second reason was to explore a further alternative explanation for the performance asymmetry: Listeners in Experiments 1-3 may have simply been more likely to interpret potentially ambiguous sounds as being from males rather than from females, thereby producing higher percentage correct results, lower latencies, and apparently greater sensitivity when the talkers were in fact males. The final experiment was therefore designed to help rule out both stimulus duration differences and response bias as possible explanations for the asymmetrical performance observed in the first three studies.

In order to do so, we used a signal detection design in which listeners heard pairs of sounds from different talkers on each trial, and judged whether the two individuals represented were of the same or different sexes. Two conditions were created - namely, hearing male-male versus malefemale pairs, and hearing female-female versus femalemale pairs. The confound between duration and number of cycles was addressed by including twice as many cycles in the male stimuli as in the female stimuli they were being compared with. Doing so created a confound for number of cycles but produced stimuli of approximately equal duration. In addition, using a signal detection procedure allowed possible response bias to be measured separately from sensitivity. In same-different designs, an overall tendency to favor "same" responses is measured as positive bias, whereas a tendency to respond "different" is measured as a negative bias (Macmillan \& Creelman, 1991). If bias did in fact play an important role in Experiments 1-3, we expected Experiment 4 to reveal more positive bias outcomes for participants discriminating male-male versus male-female pairs than for those hearing female-female versus female-male combinations.

\section{Method}

Participants. There were 28 participants ( 11 males, 17 females). The data from 2 additional male listeners were excluded, with one falling asleep during testing and the other failing to use both index fingers when responding.

Stimuli. A subset of the / $/ \varepsilon$ / stimuli from Experiment 1 were used, including sounds from 6 different males and 6 different females. The talkers were represented by 3 stimuli each, including two-, three-, and four-cycle male sounds and four-, six-, and eight-cycle female sounds. Same-sex pairings were matched on number of cycles, whereas different-sex pairings matched male sounds with female versions having twice as many cycles. Male sounds were in each case slightly shorter than the female versions they were paired with (16 vs. $18 \mathrm{msec}, 24$ vs. $27 \mathrm{msec}$, and 33 vs. $36 \mathrm{msec}$ for the shortest, intermediate, and longest segments, respectively). A set of 30 stimuli was created for each combination of cycles of a particular stimulus type, for a total of 90 male-male pairs, 90 male-female pairs, 90 female-female pairs, and 90 female-male pairs. Each individual token appeared an equal number of times within each set of 30 stimuli, thereby also balancing the representation of individual talkers within and across sets.

A complete stimulus thus consisted of two vowel segments, each of which was set to $80 \mathrm{~dB}$ RMS before being paired. After pairing, each stimulus file began with a variable amount of silence, adjusted so that the first sound ended exactly $200 \mathrm{msec}$ into the file. This sound was followed by a $600-\mathrm{msec}$ silent interval, with the second sound beginning at the $800-\mathrm{msec}$ point. As before, stimulus files ended with $100 \mathrm{msec}$ of silence.

Procedure. Participants were asked to respond "same" when they heard pairs of sounds they believed to have been produced by talkers of the same sex and "different" when they believed the sounds had been produced by talkers of different sexes. In this procedure, correctly responding "same" was considered a hit, incorrectly responding "different" was a miss, correctly responding "different" was a correct rejection, and incorrectly responding "same" was a false alarm. Data analyses were based on the differencing model for same-different designs described by Macmillan and Creelman (1991). The dependent measures were mean $d^{\prime}$ (sensitivity), median response latency, and mean $c_{\mathrm{sd}}$ (a relative bias measure that is normalized using each participant's $d^{\prime}$ outcome). The $d^{\prime}$ measure was computed from the hit and false alarm rates, median latency outcomes were tallied from hits alone, and normalized bias was calculated from hits, false alarms, and $d^{\prime}$ values.

The instructions to participants now stated that each pair of sounds had been produced by same- or different-sex talkers and that 
listeners were to judge each pair on that basis by pressing buttons labeled either Same or Different. Participants were tested in one of two conditions, either hearing male-male (same) and male-female (different) pairs in the male condition or hearing female-female and female-male pairs in the female condition. Fourteen participants were tested in each case. There were 12 practice trials, which included all stimulus combinations from 2 male and 2 female talkers, paired as appropriate to the condition being tested. These trials alternated systematically between same and different pairs, in descending order of the number of cycles.

\section{Results and Discussion}

Discrimination performance and asymmetry. Repeated measures ANOVA testing showed no overall effect of listener sex on $d^{\prime}$, latency, or $c_{\text {sd }}$, and this factor will not be considered further. Figure 5 illustrates that there were significant main effects of both condition and number of cycles on the $d^{\prime}$ and latency results. As predicted from the previous experiments, $d^{\prime}$ values were statistically higher in the male than in the female condition $[F(1,26)=6.33, p<$ $.05]$, and latencies were likewise statistically shorter in the male condition $[F(1,26), p<.05]$. Taken together, longer stimuli also produced increased $d^{\prime}$ outcomes $[F(2,52)=$ $3.88, p<.05]$, as well as longer latencies $[F(2,52)=4.09$, $p<.05]$, in comparison with shorter stimuli. Overall, these outcomes demonstrated more efficient performance when listeners responded "same" to male-male pairs than when responding "same" to female-female pairs, but also that stimulus duration had an effect.

Response bias. Bias analyses revealed strong main effects of number of cycles on $c_{\text {sd }}[F(1,84)=25.4, p<$ $.0001]$. Post hoc comparisons revealed that the effects were traceable to lower values on trials in which listeners heard pairs combining two-cycle male and/or four-cycle female stimuli $(M=-0.20, S E=0.10)$ than on trials with either three-cycle male/six-cycle female $(M=0.33$, $S E=0.10)$ or four-cycle male/eight-cycle female $(M=$ $0.41, S E=0.09)$ versions. There was also a main effect of condition on $c_{\mathrm{sd}}[F(1,84)=10.47, p<.01]$, with lower values found in the male $(M=-0.01, S E=0.09)$ than in the female $(M=0.41, S E=0.08)$ condition. In other words, although $d^{\prime}$ values were lower when participants heard either female-female or female-male pairs, participants were also more likely to choose the affirmative "same" response in this condition.

On the one hand, the results indicate that listeners were both more sensitive and likely more confident when hearing the longer stimuli, since they achieved higher $d^{\prime}$ values and were also more likely to respond with "same." This outcome seems sensible and intuitive. On the other hand, bias results indicated that participants were more likely to be affirmative in the female condition than in the male condition. This outcome is not as intuitive, at least if the bias to choose "same" is taken to reflect participant confidence. Given that $d^{\prime}$ values were lower when listeners heard female-female and female-male stimuli, it is not clear why they would have felt more sure or affirmative in this condition. The safest conclusion may therefore simply be that there was no evidence of a tendency to favor affirmative, "same" responses specifically for male stimuli.

\section{GENERAL DISCUSSION}

The results of our four experiments confirmed an a priori prediction that listeners would perform better with male than with female sounds when judging talker sex from brief vowel segments. This effect was subtle but reliable. Among the 80 total participants in the first three experiments, 60 performed more accurately with male stimuli, 13 performed more accurately with female stimuli, and 7 performed equivalently. Sixty-seven of these listeners also responded faster to male sounds, with 13 showing faster responses to female sounds.

Experiments 1-3 demonstrated that the minimum vowel duration for hearing talker sex is significantly shorter than the shortest, 50-msec stimuli tested by Whiteside (1998a). These results likely reflect both that differences between male and female $f 0$ and formant values are large and that the cues themselves are likely manifest within individual cycles of a vowel waveform. Across the three experiments, listeners could reliably determine talker sex from hearing
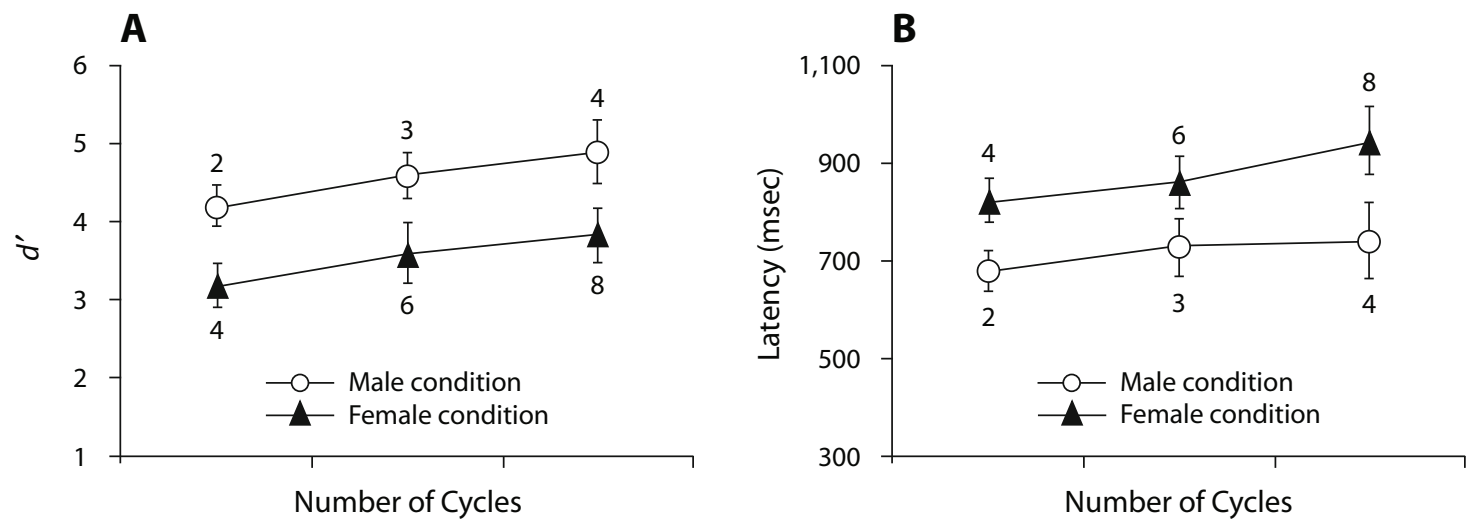

Figure 5. Same-different response data from Experiment 4 with / $/$ / sounds. (A) $d^{\prime}$ means and standard errors by number of cycles for both the male (male-male vs. male-female stimulus pairs) and female (female-female vs. female-male stimulus pairs) conditions. (B) Means of median latencies by number of cycles for each condition. Male stimuli consisted of either two, three, or four cycles, whereas female stimuli included four, six, or eight cycles. Different-sex stimuli paired two- and four-, three- and six-, and four- and eight-cycle sounds from males and females, respectively. 
approximately $14 \mathrm{msec}$ or less of a male vowel, and about $8 \mathrm{msec}$ or less of a female vowel.

\section{Asymmetry in Talker-Sex Perception}

The asymmetry of listener performance in an otherwise symmetrical classification task was specifically predicted by considering the evolutionary and developmental origins of male-female differences in vocal-tract anatomy. From this perspective, sexual selection in human evolutionary history has marked postpubertal male voices with lowered $f 0$ and formant frequencies. As a result, if one hears these distinctive features in a voice, it is a virtual certainty that the talker is an adult male. However, listeners cannot be quite so certain when those features are absent. Hearing the characteristic $f 0$ and formant values of an adult female, listeners can usually safely conclude that the talker comes from this age-sex class. However, there are also the possibilities that the talker is a prepubertal male whose characteristics resemble those of an adult female or an adult male whose vocal anatomy happened not to diverge as far from the earlier female-like state as normally occurs during development.

\section{Alternative Explanations}

Several possible alternatives for the observed results must be ruled out before accepting that the asymmetrical classification performance we observed is ultimately grounded in sexual selection and anatomical dimorphism. The confound created by having unequal stimulus durations in Experiments 1-3 is unlikely to account for the asymmetrical performance, since both controlling for number of cycles as a covariate and post hoc testing among stimuli of comparable duration mitigated against this possibility. In Experiment 4, male and female sounds were more closely matched, and in fact male sounds became slightly shorter. The performance asymmetry nonetheless still emerged strongly.

Better performance with male sounds is also unlikely to have been due to the greater acoustic diversity and larger vowel spaces reported for female speech. On the one hand, male-female differences were greatest in Experiment 2, which presented stimuli based on the point vowels $/ \mathbf{i} /, / \mathbf{u} /$, and /a/. On the other, accuracy with the two- through eightcycle male sounds in this experiment was statistically higher than accuracy with corresponding female/e/ sounds in Experiment 1 . The asymmetry also emerged strongly in Experiment 3 , which used reduced vowels that should be the least affected by an overall sex difference in dispersion.

Finally, Experiment 4 showed that the performance advantage for male stimuli was not likely due to listener response bias. Here, between-group comparisons showed a greater tendency for participants to select the affirmative, "same" response when hearing female-female versus female-male pairs than when hearing male-male versus male-female stimuli. This outcome is the opposite of what one would expect if listeners were in fact biased toward hearing ambiguous stimuli as being from males.

\section{Conclusions}

Taken together, the four experiments reported here demonstrated the predicted performance asymmetry among stimuli drawn from across the English language vowel space, including the high front (/i/), mid front $(/ \varepsilon /)$, high back (/u/), low back (/a/), and more central regions (see, e.g., Ladefoged, 2001). In the absence of support for three possible alternative explanations, we conclude that the observed sex-based performance difference mirrors the asymmetrical nature of vocal-tract anatomy and development in human males and females. Although speechstyle considerations and other cultural factors are also important in creating sex differences, discrepancies in $f 0$ and formant values are most directly traceable to underlying dimorphism in male and female vocal-tract anatomy.

Ultimately, the value of making and confirming predictions from this kind of biologically based perspective lies in the potential to illuminate the process of speech evolution. It has, for instance, long been known that indexical and phonetic cues are deeply entangled in normative speech perception processes. One striking demonstration is that of vowel normalization, the malleability of perceived vowel quality in the face of acoustic variation related to characteristics such as talker sex, vocal-tract size, and individual identity (for a review, see Johnson, 2005). Both $f 0$ values (see, e.g., Johnson, 1990b) and formant frequency cues (e.g., Ladefoged \& Broadbent, 1957) are known to produce such effects. Johnson (1990a, 1990b) argued that normalization effects are primarily mediated by perceived talker characteristics rather than by the acoustic attributes of $f 0 \mathrm{~s}$ and formants per se, and classic speech perception studies have long since demonstrated that these talker-specific dimensions play a fundamental role in the perception of vowel quality. This phonetic quality has been found to be perceptible even from extremely short vowels (see, e.g., Robinson \& Patterson, 1995; Suen \& Beddoes, 1972), and the present results have demonstrated that listeners are similarly sensitive to indexical cuing in the same kinds of sounds. Taken together, these findings support the argument that perception of talker characteristics and vowel quality are fundamentally intertwined (Johnson 1990a, 1990b, 2005; Pisoni, 1997).

\section{AUTHOR NOTE}

M.B. is now at the Department of Psychology at Vanderbilt University. This work was supported in part by NIMH Prime Award 1 R01 MH6531701A2, Subaward 8402-15235-X, an NSF POWRE award, a Georgia State University Research Initiation Grant, and the Center for Behavioral Neuroscience under the STC Program of the National Science Foundation, Agreement No. IBN-9876754. Thanks to Tom Carrell for providing programs and advice on data collection, and to Julia Albright, Laurie Belosa, Gina Cardillo, Brandon Cruse, Zeynep Eroğlu, Shaun Geer, Brian Ginsberg, Mandy Holley, Sarah Ingrid Jensen, Paul Munkholm, Erin O'Connell, Kristie Ota, Cara Starke, and Jiun Yi for research assistance. The manuscript benefited significantly from comments by T. Carrell, J. Kreiman, R. Remez, M. Pitt, and two anonymous reviewers. Experiment 3 is based on a portion of M.B.'s B.A. honors thesis at Cornell University. Correspondence concerning this article should be addressed to $\mathrm{M}$. J. Owren, Department of Psychology, Georgia State University, P.O. Box 5010, Atlanta, GA 30302-5010 (e-mail: owren@gsu.edu).

\section{REFERENCES}

Abercrombie, D. (1967). Elements of general phonetics. Chicago: Aldine. 
BACHOROWSKI, J.-A., \& OWREN, M. J. (1995). Vocal expression of emotion: Acoustic properties of speech are associated with emotional intensity and context. Psychological Science, 6, 219-224.

BACHOROWSKI, J.-A., \& OWREN, M. J. (1999). Acoustic correlates of talker sex and individual talker identity are present in a short vowel segment produced in running speech. Journal of the Acoustical Society of America, 106, 1054-1063.

Bennett, S., \& Montero-Diaz, L. (1982). Children's perception of speaker sex. Journal of Phonetics, 10, 113-121.

Boersma, P. (2001). Praat, a system for doing phonetics by computer. Glot International, 5, 341-345.

Boyd, R., \& SilK, J. B. (2000). How humans evolved (2nd ed.). New York: Norton.

Childers, D. G., \& Wu, K. (1991). Gender recognition from speech: Part II. Fine analysis. Journal of the Acoustical Society of America, 90, 1841-1856.

Coleman, R. O. (1971). Male and female voice quality and its relationship to vowel formant frequencies. Journal of Speech \& Hearing Research, 14, 565-577.

Coleman, R. O. (1976). A comparison of the contributions of two voice quality characteristics to the perception of maleness and femaleness in the voice. Journal of Speech \& Hearing Research, 19, 168-180.

Collins, S. (2000). Men's voices and women's choices. Animal Behaviour, 60, 773-780.

Deterding, D. (1997). The formants of monophthong vowels in standard southern British English pronunciation. Journal of the International Phonetic Association, 27, 47-55.

FANT, G. (1960). Acoustic theory of speech production, with calculations based on X-ray studies of Russian articulations. 's Gravenhage, The Netherlands: Mouton.

FANT, G. (1966). A note on vocal tract size factors and non-uniform F-pattern scaling. Speech Transactions Laboratory Quarterly Progress \& Status Report, 4, 22-30.

Feinberg, D. R., Jones, B. C., Little, A. C., Burt, D. M., \& Perrett, D. I. (2005). Manipulations of fundamental and formant frequencies influence the attractiveness of human male voices. Animal Behaviour, 69, 561-568.

Fitch, W. T. (2000). The evolution of speech: A comparative review. Trends in Cognitive Sciences, 4, 258-267.

Fitch, W. T., \& GiedD, J. (1999). Morphology and development of the human vocal tract: A study using magnetic resonance imaging. Journal of the Acoustical Society of America, 106, 1511-1522.

GescheIder, G. A. (1997). Psychophysics: The fundamentals (3rd ed.). Mahwah, NJ: Erlbaum.

Goldinger, S. D., Pisoni, D. B., \& Luce, P. A. (1996). Speech perception and spoken word recognition: Research and theory. In N. J. Lass (Ed.), Principles of experimental phonetics (pp. 277-327). St. Louis: Mosby.

GoldSTEIN, U. G. (1980). An articulatory model for the vocal tracts of growing children. Unpublished doctoral dissertation, Massachusetts Institute of Technology, Cambridge, MA.

Henton, C. (1995). Cross-language variation in the vowels of female and male speakers. In Proceedings of the 13th International Congress of Phonetic Sciences (Vol. 4, pp. 420-423), Stockholm.

Hillenbrand, J., Getty, L. A., Clark, M. J., \& Wheeler, K. (1995). Acoustic characteristics of American English vowels. Journal of the Acoustical Society of America, 97, 3099-3111.

Hollien, H. F. (2002). Forensic voice identification. San Diego: Academic Press.

InGEMANN, F. (1968). Identification of the speaker's sex from voiceless fricatives. Journal of the Acoustical Society of America, 44, 1142-1144.

Johnson, K. (1990a). Contrast and normalization in vowel perception. Journal of Phonetics, 18, 229-254.

JoHnson, K. (1990b). The role of perceived speaker identity in $F 0$ normalization of vowels. Journal of the Acoustical Society of America, 88, 642-654.

JoHnson, K. (2005). Speaker normalization in speech perception. In D. B. Pisoni \& R. E. Remez (Eds.), The handbook of speech perception (pp. 363-389). Malden, MA: Blackwell.

Johnson, K., \& Mullennix, J. W. (EDs.) (1997). Talker variability in speech processing. San Diego: Academic Press.

Lachs, L., McMichael, K., \& Pisoni, D. B. (2000). Speech perception and implicit memory: Evidence for detailed episodic encoding of phonetic events (Research on Spoken Language Processing, Prog- ress Report No. 24, pp. 149-167). Bloomington: Indiana University, Speech Research Laboratory.

LADEFoged, P. (1967). Three areas of experimental phonetics: Stress and respiratory activity, the nature of vowel quality, units in the perception and production of speech. Oxford: Oxford University Press.

LADEFoged, P. (2001). Vowels and consonants: An introduction to the sounds of languages. Malden, MA: Blackwell.

LAdEFoged, P., \& BROADBENT, D. E. (1957). Information conveyed by vowels. Journal of the Acoustical Society of America, 29, 98-104.

Lass, N. J., Hughes, K. R., Bowyer, M. D., Waters, L. T., \& Bourne, V. T. (1976). Speaker sex identification from voiced, whispered, and filtered isolated vowels. Journal of the Acoustical Society of America, 59, 675-678.

Lee, S., Potamianos, A., \& Narayanan, S. (1999). Acoustics of children's speech: Developmental changes of temporal and spectral parameters. Journal of the Acoustical Society of America, 105, 14551468.

Macmillan, N. A., \& Creelman, C. D. (1991). Detection theory: A user's guide. Cambridge: Cambridge University Press.

Perry, T. L., Ohde, R. N., \& Ashmead, D. H. (2001). The acoustic bases for gender identification from children's voices. Journal of the Acoustical Society of America, 109, 2988-2998.

Peterson, G. E., \& Barney, H. L. (1952). Control methods used in a study of the vowels. Journal of the Acoustical Society of America, 24, 175-184.

PISONI, D. B. (1997). Some thoughts on "normalization" in speech perception. In K. Johnson \& J. W. Mullennix (Eds.), Talker variability in speech processing (pp. 9-32). San Diego: Academic Press.

PuTs, D. A. (2005). Mating context and menstrual phase affect women's preferences for male voice pitch. Evolution \& Human Behavior, 26, 388-397.

Puts, D. A., Gaulin, S. J. C., \& Verdolini, K. (2006). Dominance and the evolution of sexual dimorphism in human voice pitch. Evolution \& Human Behavior, 27, 283-296.

Rendall, D., Kollias, S., Ney, C., \& Lloyd, P. (2005). Pitch $\left(F_{0}\right)$ and formant profiles of human vowels and vowel-like baboon grunts: The role of vocalizer body size and voice-acoustic allometry. Journal of the Acoustical Society of America, 117, 944-955.

Robinson, K., \& Patterson, R. D. (1995). The stimulus duration required to identify vowels, their octave, and their pitch chroma. Journal of the Acoustical Society of America, 98, 1858-1865.

Rose, P. (2002). Forensic speaker identification. London: Taylor \& Francis.

ScHWARZ, M. F. (1968). Identification of speaker sex from isolated voiceless fricatives. Journal of the Acoustical Society of America, $\mathbf{4 3}$, 1178-1179.

Schwarz, M. F., \& Rine, H. E. (1968). Identification of speaker sex from isolated whispered vowels. Journal of the Acoustical Society of America, 44, 1736-1737.

Suen, C. Y., \& Beddoes, M. P. (1972). Discrimination of vowel sounds of very short duration. Perception \& Psychophysics, 11, 417-419.

Titze, I. R. (1989). Physiologic and acoustic differences between male and female voices. Journal of the Acoustical Society of America, 85, 1699-1707.

Titze, I. R. (1994). Principles of voice production. Englewood Cliffs, NJ: Prentice Hall.

Traunmüller, H. (1988). Paralinguistic variation and invariance in the characteristic frequencies of vowels. Phonetica, 45, 1-29.

Traunmüller, H. (1997). Perception of speaker sex, age, and vocal effort. Phonum, 4, 183-186.

Whiteside, S. P. (1998a). Identification of speaker's sex: A study of vowels. Perceptual \& Motor Skills, 86, 579-584.

Whiteside, S. P. (1998b). Identification of speaker's sex: A fricative study. Perceptual \& Motor Skills, 86, 587-591.

Whiteside, S. P. (2001). Sex-specific fundamental and formant frequency patterns in a cross-sectional study. Journal of the Acoustical Society of America, 110, 464-478.

Wu, K., \& ChILders, D. G. (1991). Gender recognition from speech: Part I. Coarse analysis. Journal of the Acoustical Society of America, 90, 1828-1840.

(Manuscript received July 7, 2004; revision accepted for publication January 12, 2007.) 